\title{
Fault detection for continuous-time switched systems under asynchronous switching
}

\author{
Dongsheng $\mathrm{Du}^{1,2}$, Bin Jiang ${ }^{1, *, \dagger}$, Peng Shi ${ }^{3,4,5}$ and Hamid Reza Karimi ${ }^{6}$ \\ ${ }^{1}$ College of Automation Engineering, Nanjing University of Aeronautics and Astronautics, Nanjing 210016, \\ Jiangsu, China \\ ${ }^{2}$ School of Science, Huaihai Institute of Technology, 59 Cangwu Road, Lianyungang 222005, Jiangsu, China \\ ${ }^{3}$ Department of Computing and Mathematical Sciences, University of Glamorgan, Pontypridd, CF37 1DL, UK ${ }^{4}$ School \\ of Engineering and Science, Victoria University, Melbourne 8001 Vic, Australia \\ ${ }^{5}$ School of Electrical and Electronic Engineering, The University of Adelaide, Adelaide SA 5005, Australia ${ }^{6}$ Department \\ of Engineering Faculty of Engineering and Science, University of Agder, N-4898 Grimstad, Norway
}

\begin{abstract}
SUMMARY
In this paper, the problem of fault detection for continuous-time switched systems under asynchronous switching is investigated. The designed fault detection filter is assumed to be asynchronous with the original systems. Attention is focused on designing a fault detection filter such that the estimation error between the residual and the fault is minimized in the sense of $H_{\infty}$ norm. By employing piecewise Lyapunov function and average dwell time techniques, a sufficient condition for the existence of such a filter is exploited in terms of certain linear matrix inequalities. Finally, an example of a switched electrical circuit is provided to illustrate the effectiveness of the proposed approach.
\end{abstract}

Received 5 January 2012; Revised 21 October 2012; Accepted 13 December 2012

\section{INTRODUCTION}

In recent years, the process of fault detection and isolation (FDI) for dynamic systems has been of considerable interest, and fruitful model-based fault detection results have been obtained in several excellent papers [1-9] and books [10,11]. Among these model-based approaches of FDI, the basic idea is to use state observer or filter to construct a residual signal and, on the basis of this, to determine a residual evaluation function to compare with a predefined threshold. When the residual evaluation function has a value larger than the threshold, an alarm of faults is generated. On the other hand, it is well known that control inputs, unavoidable unknown inputs, and faults are coupled in many industrial systems, which are potential sources of false alarm. This means that FDI systems have to be robust to control inputs and unknown inputs and at the same time enhance the sensitivity to the faults. Therefore, it is of great significance to design a model-based fault detection system. In reviewing the development of the theories and techniques for different FDI system designs, a number of results have been obtained in designing FDI system. For examples, in [12], an $H_{\infty}$ fil-ter formulation of robust FDI has been considered for uncertain LTI systems. The issue of $H_{\infty}$ fault detection filter design for linear discrete-time systems with multiple time delays is investigated in [5].

\footnotetext{
*Correspondence to: Bin Jiang, College of Automation Engineering, Nanjing University of Aeronautics and Astronautics, Nanjing 210016, Jiangsu, China.

†E-mail: binjiang@nuaa.edu.cn
} 
On another research direction, switched systems have attracted increasing attention in the literature of control problems due to their great significance in theory and practical applications. Switched systems belong to hybrid systems, which consist of a class of subsystems and a switching signal. The switching signal specifies which subsystem will be activated along the trajectory at each instant of time. Presently, many achievements have been achieved on the control of switched system [13-21]. Especially, in recent years, it is a hot topic to study the problem of fault diagnosis and fault tolerant control for switched systems. For examples, fault detection problem is separately considered in [22-24] for discrete case and in [25] for continuous case. Fault diagnosis for continuous-time switched system was investigated in [26], whereas [27] studied fault tolerant control problem for continuous-time switched systems. However, all the aforementioned literatures assume that the observer or filter is synchronous with the original systems. Actually, it often takes time to identify the system. Therefore, the phenomenon of asynchronous switching generally exists. Moreover, the developed methods of fault detection for switched systems under synchronous switching are no longer suitable to solve asynchronous switching case. New technique should be contrived to solve this issue. In [28], the maximum dwell time technique is used to deal with reliable control problem for switched nonlinear systems under asynchronous switching. A less conservative approach is still under research. Until now, to the best of our knowledge, the problem of fault detection for continuous-time switched systems under asynchronous switching has not been considered yet, which motivates us to study this interesting and practical issue.

In this paper, the problem of fault detection for continuous-time switched systems under asynchronous switching is investigated. Firstly, by using piecewise Lyapunov function and average dwell time technique, a sufficient condition for the $H_{\infty}$ fault detection filter is exploited in the formation of LMI. Then, based on the obtained condition, a desired fault detection filter is constructed. Finally, to demonstrate the feasibility and effectiveness of the proposed method, a simulation example of a switched electrical circuit is included.

The rest of this paper is organized as follows. In Section 2, system descriptions and problem formulation are presented. A sufficient condition on the existence of a fault detection filter for continuous-time switched systems is derived in terms of LMIs, and the parameters of the desired filter are constructed by solving the corresponding LMIs in Section 3. To demonstrate the validity of the proposed approach, an example is given in Section 4 that is followed by a conclusion in Section 5.

\section{PROBLEM FORMULATION}

Consider the following class of continuous-time switched systems:

$$
\left\{\begin{array}{l}
\dot{x}(t)=A_{\sigma(t)} x(t)+B_{1 \sigma(t)} d(t)+D_{1 \sigma(t)} f(t) \\
y(t)=C_{\sigma(t)} x(t)+B_{2 \sigma(t)} d(t)+D_{2 \sigma(t)} f(t)
\end{array}\right.
$$

where $x(t) \in \mathbb{R}^{n}$ is the output vector, $y(t) \in \mathbb{R}^{m}$ is the output vector, $d(t) \in \mathbb{R}^{p}$ is the unknown input vector (including disturbance, noise, or structured model uncertainty), and $f(t) \in \mathbb{R}^{q}$ is the fault. $\sigma(t):[0,+\infty) \longrightarrow \psi=\{1, \cdots, N\}$ is the switching signal that specifies which subsystem will be activated, and $N$ denotes the number of subsystems. In this paper, we assume that the switching signal $\sigma(t)$ is time-dependent, that is, $\sigma(t):\left\{\left(t_{0}, \sigma\left(t_{0}\right)\right),\left(t_{1}, \sigma\left(t_{1}\right)\right), \cdots\right\}$, where $t_{0}$ denotes the initial time, and $t_{k}$ denotes the $k$ th switching instant. $A_{\sigma(t)}, B_{1 \sigma(t)}, B_{2 \sigma(t)}, C_{\sigma(t)}, D_{1 \sigma(t)}$, and $D_{2 \sigma(t)}$ are constant matrices with appropriate dimensions for all $\sigma(t) \in \psi$. We denote the matrices associated with $\sigma(t)=i$ by $A_{\sigma(t)}=A_{i}, B_{1 \sigma(t)}=B_{1 i}, B_{2 \sigma(t)}=B_{2 i}, C_{\sigma(t)}=C_{i}, D_{1 \sigma(t)}=D_{1 i}$, and $D_{2 \sigma(t)}=D_{2 i}$.

Actually, there inevitably exists asynchronous switching between the filter and the original system in actual operation. Therefore, we suppose the $i$ th subsystem is activated at the switching instant $t_{k-1}$, the $j$ th subsystem is activated at the switching instant $t_{k}$, and the corresponding switching filter is activated at the switching instants $t_{k-1}+\Delta_{k-1}$ and $t_{k}+\Delta_{k}$, respectively. Owing to asynchronous switching, the switching instant of the fault detection filter corresponding to $j$ th subsystem is $t_{k}+\Delta_{k}$, and then, there exists a matched period at time interval $\left[t_{k-1}+\Delta_{k-1}, t_{k}\right)$ and a 


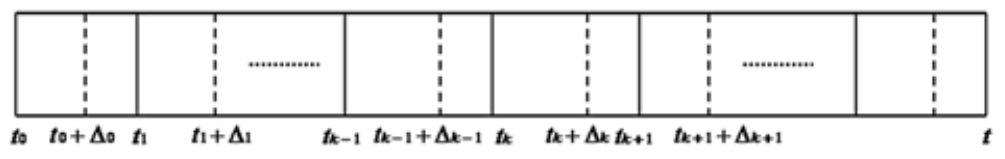

Figure 1. Diagram of asynchronous switching.

mismatched period at time interval $\left[t_{k}, t_{k}+\Delta_{k}\right)$. The case that the switching instants of the filter experience lags with respect to those of the system can be described by Figure 1.

For the purpose of residual generation, the following fault detection filter is constructed as a residual generator. For convenience, there we use $\sigma^{\prime}(t)$ to denote the switching signal of the fault detection filter:

$$
\left\{\begin{array}{l}
\dot{\hat{x}}(t)=A_{f \sigma^{\prime}(t)} \hat{x}(t)+B_{f \sigma^{\prime}(t)} y(t) \\
r(t)=C_{f \sigma^{\prime}(t)} \hat{x}(t)+D_{f \sigma^{\prime}(t)} y(t)
\end{array}\right.
$$

where $\hat{x}(t) \in \mathbb{R}^{n}$ is the filter's state and $r(t) \in \mathbb{R}^{q}$ is the residual signal. $A_{f \sigma^{\prime}(t)}, B_{f \sigma^{\prime}(t)}, C_{f \sigma^{\prime}(t)}$, and $D_{f \sigma^{\prime}(t)}$ are filter parameters to be determined.

Denoting $e(t)=r(t)-f(t)$ and augmenting state vector $\tilde{x}(t)=\left[\begin{array}{cc}x^{T}(t) & \hat{x}^{T}(t)\end{array}\right]^{T}, \omega(t)=$ $\left[\begin{array}{ll}d^{T}(t) & f^{T}(t)\end{array}\right]^{T}$. When $t \in\left[t_{0}, t_{1}\right) \cup\left[t_{k-1}+\Delta_{k-1}, t_{k}\right), k=2,3,4, \ldots$, we obtain the augmented system as follows:

$$
\Sigma: \quad\left\{\begin{array}{l}
\dot{\tilde{x}}(t)=\widetilde{A}_{i} \tilde{x}(t)+\widetilde{B}_{i} \omega(t) \\
e(t)=\widetilde{C}_{i} \tilde{x}(t)+\widetilde{D}_{i} \omega(t)
\end{array}\right.
$$

When $t \in\left[t_{k}, t_{k}+\Delta_{k}\right), k=1,2,3, \ldots$, the augmented system is represented as follows:

$$
\Sigma^{\prime}: \quad\left\{\begin{array}{l}
\dot{\tilde{x}}(t)=\widetilde{A}_{i j} \tilde{x}(t)+\widetilde{B}_{i j} \omega(t) \\
e(t)=\widetilde{C}_{i j} \tilde{x}(t)+\widetilde{D}_{i j} \omega(t)
\end{array}\right.
$$

where

$$
\left\{\begin{aligned}
\widetilde{A}_{i} & =\left[\begin{array}{cc}
A_{i} & 0 \\
B_{f i} C_{i} & A_{f i}
\end{array}\right], \widetilde{B}_{i}=\left[\begin{array}{cc}
B_{1 i} & D_{1 i} \\
B_{f i} B_{2 i} & B_{f i} D_{2 i}
\end{array}\right], \widetilde{C}_{i}=\left[\begin{array}{cc}
D_{f i} C_{i} & C_{f i}
\end{array}\right], \\
\widetilde{D}_{i} & =\left[\begin{array}{cc}
D_{f i} B_{2 i} & D_{f i} D_{2 i}-I
\end{array}\right] \\
\widetilde{A}_{i j} & =\left[\begin{array}{cc}
A_{j} & 0 \\
B_{f i} C_{j} & A_{f i}
\end{array}\right], \widetilde{B}_{i j}=\left[\begin{array}{cc}
B_{1 j} & D_{1 j} \\
B_{f i} B_{2 j} & B_{f i} D_{2 j}
\end{array}\right], \widetilde{C}_{i j}=\left[\begin{array}{cc}
D_{f i} C_{j} & C_{f i}
\end{array}\right], \\
\widetilde{D}_{i j} & =\left[\begin{array}{ll}
D_{f i} B_{2 j} & D_{f i} D_{2 j}-I
\end{array}\right] .
\end{aligned}\right.
$$

Now, the problem of fault detection filter design can be formulated as an $H_{\infty}$ filter problem: to develop filter (2) for system (1) such that the augmented system $\Sigma\left(\right.$ or $\left.\Sigma^{\prime}\right)$ is stable when $\omega(t)=0$ and under zero-initial condition, the minimum of $\gamma$ is made small in the feasibility of

$$
\sup _{\|\omega(t)\|_{2} \neq 0}\left\{\frac{\|e(t)\|_{2}}{\|\omega(t)\|_{2}}\right\}<\gamma, \quad \gamma>0
$$

After designing the residual generator, the last step to a successful fault detection is the residual evaluation stage including an evaluation function and a threshold. In this paper, the threshold $J_{\text {th }}$ and residual evaluation function $J_{\mathrm{r}}(L)$ are selected as

$$
\begin{gathered}
J_{\mathrm{r}}(L)=\|r(t)\|_{2}=\left(\int_{0}^{L} r^{T}(\varsigma) r(\varsigma) \mathrm{d} \varsigma\right)^{\frac{1}{2}} \\
J_{\mathrm{th}}=\sup _{d \in l_{2}, f=0}\|r(t)\|_{2}
\end{gathered}
$$


where $L$ is the evaluation time steps. On the basis of this, the occurrence of faults can be detected by comparing $J_{\mathrm{r}}(L)$ and $J_{\mathrm{th}}$ according the following test:

$$
\begin{gathered}
J_{\mathrm{r}}(L)>J_{\text {th }} \Longrightarrow \text { with faults } \Longrightarrow \text { alarm } \\
J_{\mathrm{r}}(L) \leqslant J_{\text {th }} \Longrightarrow \text { no faults }
\end{gathered}
$$

Remark 1

The fault detection filter design for continuous-time switched systems with time-varying delay has been exploited in [25]. However, the designed fault detection filter must be matched with the original systems. In this note, the mismatched case is considered, and the obtained results can be directly extended to delay case and parameter uncertain case.

Definition 1 ([29])

For any switching signal $\sigma(t)$ and any $t_{2}>t_{1}>0$, let $N_{\sigma}(\tau, t)$ denote the number of switchings of $\sigma(t)$ on an interval $\left(t_{1}, t_{2}\right)$. If

$$
N_{\sigma}(\tau, t) \leqslant N_{0}+\frac{t_{2}-t_{1}}{\tau_{a}}
$$

holds for a given $N_{0} \geqslant 0$ and $\tau_{a}>0$, then the constant $\tau_{a}$ is called the average dwell time and $N_{0}$ the chattering bound.

Lemma 1 ([30])

If there exist functions $\phi(t)$ and $v(t)$ satisfying

$$
\dot{\phi}(t) \leqslant-\zeta \phi(t)+\kappa v(t)
$$

then

$$
\phi(t) \leqslant e^{-\zeta\left(t-t_{0}\right)} \phi\left(t_{0}\right)+\kappa \int_{0}^{t-t_{0}} e^{-\zeta \tau} v(t-\tau) \mathrm{d} \tau
$$

\section{FAULT DETECTION FILTER DESIGN}

\section{1. $H_{\infty}$ performance analysis}

Theorem 1

Given constants $\alpha>0, \beta>0, \mu_{1} \geqslant 1$, and $\mu_{2} \geqslant 1$, if there exist matrices $P_{i}>0, P_{i j}>0$, for $i \neq j, i, j \in N$, such that

$$
\begin{gathered}
P_{j} \leqslant \mu_{1} P_{i j}, \quad P_{i j} \leqslant \mu_{2} P_{i} ; \\
{\left[\begin{array}{ccc}
\widetilde{A}_{i}^{T} P_{i}+P_{i} \widetilde{A}_{i}+\alpha P_{i} & P_{i} \widetilde{B}_{i} & \widetilde{C}_{i}^{T} \\
* & -\gamma^{2} I & \widetilde{D}_{i}^{T} \\
* & * & -I
\end{array}\right]<0} \\
{\left[\begin{array}{ccc}
\widetilde{A}_{i j}^{T} P_{i j}+P_{i j} \widetilde{A}_{i j}-\beta P_{i j} & P_{i j} \widetilde{B}_{i j} & \widetilde{C}_{i j}^{T} \\
* & -\gamma^{2} I & \widetilde{D}_{i j}^{T} \\
* & * & -I
\end{array}\right]<0}
\end{gathered}
$$

then the system $\Sigma\left(\right.$ or $\left.\Sigma^{\prime}\right)$ is asymptotically stable with $H_{\infty}$ performance $\gamma$ for any switching signal with average dwell time satisfying

$$
\tau_{a}>\tau_{a}^{*}=\frac{\ln \left(\mu_{1} \mu_{2}\right)}{\zeta^{*}}, \quad \frac{T^{-}\left(t_{0}, t\right)}{T^{+}\left(t_{0}, t\right)} \geqslant \frac{\beta+\zeta^{*}}{\alpha-\zeta^{*}}, \quad 0<\zeta^{*}<\alpha
$$

where $T^{-}\left(t_{0}, t\right)$ or $T^{+}\left(t_{0}, t\right)$ denotes the total matched or mismatched period during $\left[t_{0}, t\right]$. 
Proof

We first establish the stability of the the system $\Sigma\left(\right.$ or $\left.\Sigma^{\prime}\right)$. To this end, assume that $\omega(t)=0$. When $t \in\left[t_{0}, t_{1}\right) \cup\left[t_{k-1}+\Delta_{k-1}, t_{k}\right), k=2,3,4, \ldots$, the augmented system can be written as in (3). Consider the following Lyapunov function:

$$
V_{i}(t)=\tilde{x}^{T}(t) P_{i} \tilde{x}(t)
$$

Then, along the trajectory of system (3), we have

$$
\begin{aligned}
\dot{V}_{i}(t)+\alpha V_{i}(t) & =\dot{\tilde{x}}^{T}(t) P_{i} \tilde{x}(t)+\tilde{x}^{T}(t) P_{i} \dot{\tilde{x}}(t)+\alpha \tilde{x}^{T}(t) P_{i} \tilde{x}(t) \\
& =\tilde{x}^{T}(t)\left(\widetilde{A}_{i}^{T} P_{i}+P_{i} \widetilde{A}_{i}+\alpha P_{i}\right) \tilde{x}(t)
\end{aligned}
$$

then, by (10), we obtain

$$
\dot{V}_{i}(t)+\alpha V_{i}(t) \leqslant 0
$$

it follows that

$$
\dot{V}_{i}(t) \leqslant-\alpha V_{i}(t)
$$

then, during the matched period, $V_{i}(t)$ satisfy

$$
V_{i}(t) \leqslant\left\{\begin{array}{lc}
V_{i}\left(t_{0}\right) e^{-\alpha\left(t-t_{0}\right)}, & t_{0} \leqslant t<t_{1} \\
V_{i}\left(t_{k-1}+\Delta_{k-1}\right) e^{-\alpha\left(t-t_{k-1}-\Delta_{k-1}\right)}, & t_{k-1}+\Delta_{k-1} \leqslant t<t_{k}, k=2,3, \ldots
\end{array}\right.
$$

When $t \in\left[t_{k}, t_{k}+\Delta_{k}\right), k=1,2,3, \ldots$, the augmented system can be written as in (4). Consider the following Lyapunov function:

$$
V_{i j}(t)=\tilde{x}^{T}(t) P_{i j} \tilde{x}(t)
$$

Then, along the trajectory of system (4), we have

$$
\begin{aligned}
\dot{V}_{i j}(t)-\beta V_{i j}(t) & =\dot{\tilde{x}}^{T}(t) P_{i j} \tilde{x}(t)+\tilde{x}^{T}(t) P_{i j} \dot{\tilde{x}}(t)-\beta \tilde{x}^{T}(t) P_{i j} \tilde{x}(t) \\
& =\tilde{x}^{T}(t)\left(\widetilde{A}_{i j}^{T} P_{i j}+P_{i j} \widetilde{A}_{i j}-\beta P_{i j}\right) \tilde{x}(t)
\end{aligned}
$$

then, by (10), we obtain

$$
\dot{V}_{i j}(t)-\beta V_{i j}(t) \leqslant 0
$$

it follows that

$$
\dot{V}_{i j}(t) \leqslant \beta V_{i}(t)
$$

then, during the unmatched period, $V_{i j}(t)$ satisfy

$$
V_{i j}(t) \leqslant V_{i j}\left(t_{k}\right) e^{\beta\left(t-t_{k}\right)}, \quad t_{k} \leqslant t<t_{k}+\Delta_{k}, k=1,2,3, \ldots
$$

Let $t_{1}, t_{2}, \ldots, t_{k}, \ldots$ denote the switching instant of $\sigma(t)$ over the interval $\left[t_{0}, t\right]$. Consider the following piecewise Lyaounov functional candidate for system $\Sigma$ in (3) and (4):

$$
V(t)= \begin{cases}V_{i}(t)=\tilde{x}^{T}(t) P_{i} \tilde{x}(t), & t \in\left[t_{0}, t_{1}\right) \cup\left[t_{k-1}+\Delta_{k-1}, t_{k}\right), k=2,3,4, \ldots \\ V_{i j}(t)=\tilde{x}^{T}(t) P_{i j} \tilde{x}(t), & t \in\left[t_{k}, t_{k}+\Delta_{k}\right), k=1,2,3, \ldots\end{cases}
$$


When $t \in\left[t_{k}, t_{k}+\Delta_{k}\right), k=1,2,3, \ldots$, and with the condition in (9), (18) and (24), we have

$$
\begin{aligned}
V(t)= & V_{\sigma^{\prime}\left(t_{k-1}+\Delta_{k-1}\right) \sigma\left(t_{k}\right)}(t) \leqslant V_{\sigma^{\prime}\left(t_{k-1}+\Delta_{k-1}\right) \sigma\left(t_{k}\right)}\left(t_{k}\right) e^{\beta\left(t-t_{k}\right)} \\
\leqslant & \mu_{2} V_{\sigma\left(t_{k-1}\right)}\left(t_{k}^{-}\right) e^{\beta\left(t-t_{k}\right)} \\
\leqslant & \mu_{2} V_{\sigma\left(t_{k-1}\right)}\left(t_{k-1}+\Delta_{k-1}\right) e^{\beta\left(t-t_{k}\right)-\alpha\left(t-t_{k-1}-\Delta_{k-1}\right)} \\
\leqslant & \mu_{2}\left(\mu_{1} V_{\sigma^{\prime}\left(t_{k-2}+\Delta_{k-2}\right) \sigma\left(t_{k-1}\right)}\left[\left(t_{k-1}+\Delta_{k-1}\right)^{-}\right]\right) e^{\beta\left(t-t_{k}\right)-\alpha\left(t-t_{k-1}-\Delta_{k-1}\right)} \\
= & \mu_{2}^{N_{\sigma(t)}\left(t_{k-1}, t\right)} \mu_{1}^{\left.N_{\sigma^{\prime}(t)}\left(t_{k-2}+\Delta_{k-2}\right), t\right)} V_{\sigma^{\prime}\left(t_{k-2}+\Delta_{k-2}\right) \sigma\left(t_{k-1}\right)}\left(t_{k-1}\right) e^{\beta\left(t-t_{k}\right)-\alpha\left(t-t_{k-1}-\Delta_{k-1}\right)} \\
\leqslant & \cdots \ldots \ldots \ldots \ldots \ldots \ldots \ldots \ldots \\
\leqslant & \mu_{2}^{N_{\sigma(t)}\left(t_{0}, t\right)} \mu_{1}^{N_{\sigma^{\prime}(t)}\left(t_{0}, t\right)} V_{\sigma\left(t_{0}\right)}\left(t_{0}\right) e^{-\alpha\left(t_{1}-t_{0}\right)} e^{\beta\left(t-t_{k}+\Delta_{k-1}+\cdots+\Delta_{1}\right)} \\
& \times e^{-\alpha\left[\left(t_{k}-t_{k-1}-\Delta_{k-1}\right)+\left(t_{k-1}-t_{k-2}-\Delta_{k-2}\right)+\cdots+\left(t_{2}-t_{1}-\Delta_{1}\right)\right]} \\
= & \mu_{2}^{N_{\sigma(t)}\left(t_{0}, t\right)} \mu_{1}^{N_{\sigma(t)}\left(t_{0}, t\right)-1} V_{\sigma\left(t_{0}\right)}\left(t_{0}\right) e^{\beta\left(t-t_{k}+\Delta_{k-1}+\cdots+\Delta_{1}\right)} \\
& \times e^{-\alpha\left[\left(t_{k}-t_{k-1}-\Delta_{k-1}\right)+\left(t_{k-1}-t_{k-2}-\Delta_{k-2}\right)+\cdots+\left(t_{2}-t_{1}-\Delta_{1}\right)+\left(t_{1}-t_{0}\right)\right]}
\end{aligned}
$$

By (12), we have

$$
\beta T^{+}\left(t_{0}, t\right)-\alpha T^{-}\left(t_{0}, t\right) \leqslant-\zeta^{*}\left(t-t_{0}\right)
$$

then (26) and (27) imply that

$$
\begin{aligned}
V(t) & \leqslant\left(\mu_{1} \mu_{2}\right)^{N_{\sigma(t)}\left(t_{0}, t\right)} \mu_{1}^{-1} V_{\sigma\left(t_{0}\right)}\left(t_{0}\right) e^{\beta T^{+}\left(t_{0}, t\right)-\alpha T^{-}\left(t_{0}, t\right)} \\
& \leqslant\left(\mu_{1} \mu_{2}\right)^{N_{0}+\frac{t-t_{0}}{\tau_{a}}} \mu_{1}^{-1} V_{\sigma\left(t_{0}\right)}\left(t_{0}\right) e^{-\zeta^{*}\left(t-t_{0}\right)} \\
& =\mu_{1}^{-1} V_{\sigma\left(t_{0}\right)}\left(t_{0}\right) e^{N_{0} \ln \left(\mu_{1} \mu_{2}\right)} e^{-\left(\zeta^{*}-\frac{\ln \left(\mu_{1} \mu_{2}\right)}{\tau_{a}}\right)\left(t-t_{0}\right)}
\end{aligned}
$$

Therefore, if the average dwell time satisfies (12), we conclude $V(t)$ converges to zero as $t \longrightarrow \infty$. Then, the stability of system $\Sigma\left(\right.$ or $\left.\Sigma^{\prime}\right)$ can be deduced.

For any nonzero $\omega(t) \in L_{2}[0, \infty)$ and zero initial condition $\tilde{x}(0)=0$. When $t \in\left[t_{0}, t_{1}\right) \cup\left[t_{k-1}+\right.$ $\left.\Delta_{k-1}, t_{k}\right), k=2,3,4, \ldots$, the augmented system can be written as in (3). Consider the Lyapunov function as in (13) and set $\Gamma(t)=-e^{T}(t) e(t)+\gamma^{2} \omega^{T}(t) \omega(t)$, one has

$$
\dot{V}_{i}(t)+\alpha V_{i}(t)-\Gamma(t)=\xi^{T}(t) \Theta_{i} \xi(t)
$$

where $\xi(t)=\left[\begin{array}{ll}\tilde{x}^{T}(t) & \omega^{T}(t)\end{array}\right]^{T}, \Theta_{i}=\left[\begin{array}{cc}\widetilde{A}_{i}^{T} P_{i}+P_{i} \widetilde{A}_{i}+\alpha P_{i}+\widetilde{C}_{i}^{T} \widetilde{C}_{i} & P_{i} \widetilde{B}_{i}+\widetilde{C}_{i}^{T} \widetilde{D}_{i} \\ \widetilde{B}_{i}^{T} P_{i}+\widetilde{D}_{i}^{T} \widetilde{C}_{i} & -\gamma^{2} I+\widetilde{D}_{i}^{T} \widetilde{D}_{i}\end{array}\right]$.

By (10), it follows that

$$
\dot{V}_{i}(t)<-\alpha V_{i}(t)+\Gamma(t)
$$

From Lemma 1, one has

$$
V_{i}(t) \leqslant\left\{\begin{array}{l}
e^{-\alpha\left(t-t_{0}\right)} V_{i}\left(t_{0}\right)+\int_{t_{0}}^{t} e^{-\alpha(t-s)} \Gamma^{T}(s) \Gamma(s) \mathrm{d} s, \quad t_{0} \leqslant t<t_{1} \\
e^{-\alpha\left(t-t_{k-1}-\Delta_{k-1}\right)} V_{i}\left(t_{k-1}+\Delta_{k-1}\right) \\
\quad+\int_{t_{k-1}+\Delta_{k-1}}^{t} e^{-\alpha(t-s)} \Gamma^{T}(s) \Gamma(s) \mathrm{d} s, t_{k-1}+\Delta_{k-1} \leqslant t<t_{k}, \\
k=2,3, \ldots
\end{array}\right.
$$


When $t \in\left[t_{k}, t_{k}+\Delta_{k}\right), k=1,2,3, \ldots$, the augmented system can be written as in (4). Consider the Lyapunov function as in (19), one has

where $\Theta_{i j}=\left[\begin{array}{cc}\dot{V}_{i}(t)-\beta V_{i}(t)-\Gamma(t)=\xi^{T}(t) \Theta_{i j} \xi(t) \\ \widetilde{A}_{i j}^{T} P_{i j}+P_{i j} \widetilde{A}_{i j}-\beta P_{i j}+\widetilde{C}_{i j}^{T} \widetilde{C}_{i j} & P_{i j} \widetilde{B}_{i j}+\widetilde{C}_{i j}^{T} \widetilde{D}_{i j} \\ \widetilde{B}_{i j}^{T} P_{i j}+\widetilde{D}_{i j}^{T} \widetilde{C}_{i j} & -\gamma^{2} I+\widetilde{D}_{i j}^{T} \widetilde{D}_{i j}\end{array}\right]$.

By (11), it follows that

$$
\dot{V}_{i j}(t)<\beta V_{i j}(t)+\Gamma(t)
$$

From Lemma 1, one has

$$
V_{i j}(t) \leqslant e^{\beta\left(t-t_{k}\right)} V_{i j}\left(t_{k}\right)+\int_{t_{k}}^{t} e^{\beta(t-s)} \Gamma^{T}(s) \Gamma(s) \mathrm{d} s, \quad t_{k} \leqslant t<t_{k}+\Delta_{k}
$$

Consider the piecewise Lyapunov function as in (25), when $t \in\left[t_{k}, t_{k}+\Delta_{k}\right), k=1,2,3, \ldots$, it follows from (31) and (34) that

$$
\begin{aligned}
& V(t) \leqslant V_{\sigma^{\prime}\left(t_{k-1}+\Delta_{k-1}\right) \sigma\left(t_{k}\right)}\left(t_{k}\right) e^{\beta T^{+}\left(t_{k}, t\right)-\alpha T^{-}\left(t_{k}, t\right)}+\int_{t_{k}}^{t} e^{\beta T^{+}(s, t)-\alpha T^{-}(s, t)} \Gamma^{T}(s) \Gamma(s) \mathrm{d} s \\
& \leqslant \mu_{2} V_{\sigma\left(t_{k-1}\right)}\left(t_{k}^{-}\right) e^{\beta T^{+}\left(t_{k}, t\right)-\alpha T^{-}\left(t_{k}, t\right)}+\int_{t_{k}}^{t} e^{\beta T^{+}(s, t)-\alpha T^{-}(s, t)} \Gamma^{T}(s) \Gamma(s) \mathrm{d} s \\
& \leqslant \mu_{2} V_{\sigma\left(t_{k-1}\right)}\left(t_{k-1}+\Delta_{k-1}\right) e^{\beta T^{+}\left(t_{k-1}+\Delta_{k-1}, t\right)-\alpha T^{-}\left(t_{k-1}+\Delta_{k-1}, t\right)} \\
& +\mu_{2} \int_{t_{k-1}+\Delta_{k-1}}^{t} e^{\beta T^{+}(s, t)-\alpha T^{-}(s, t)} \Gamma^{T}(s) \Gamma(s) \mathrm{d} s+\int_{t_{k}}^{t} e^{\beta T^{+}(s, t)-\alpha T^{-}(s, t)} \Gamma^{T}(s) \Gamma(s) \mathrm{d} s \\
& \leqslant \mu_{2} \mu_{1} V_{\sigma^{\prime}\left(t_{k-2}+\Delta_{k-2}\right) \sigma\left(t_{k-1}\right)}\left(t_{k-1}\right) e^{\beta T^{+}\left(t_{k-1}, t\right)-\alpha T^{-}\left(t_{k-1}, t\right)} \\
& +\mu_{2} \mu_{1} \int_{t_{k-1}}^{t_{k-1}+\Delta_{k-1}} e^{\beta T^{+}(s, t)-\alpha T^{-}(s, t)} \Gamma^{T}(s) \Gamma(s) \mathrm{d} s \\
& +\mu_{2} \int_{t_{k-1}+\Delta_{k-1}}^{t_{k}} e^{\beta T^{+}(s, t)-\alpha T^{-}(s, t)} \Gamma^{T}(s) \Gamma(s) \mathrm{d} s+\int_{t_{k}}^{t} e^{\beta T^{+}(s, t)-\alpha T^{-}(s, t)} \Gamma^{T}(s) \Gamma(s) \mathrm{d} s \\
& \leqslant \cdots \cdots \cdots \cdots \cdots \\
& \leqslant \mu_{2}^{N_{\sigma(t)}\left(t_{0}, t\right)} \mu_{1}^{N_{\sigma^{\prime}(t)}\left(t_{0}, t\right)} V_{\sigma\left(t_{0}\right)}\left(t_{0}\right) e^{\beta T^{+}\left(t_{0}, t\right)-\alpha T^{-}\left(t_{0}, t\right)} \\
& +\mu_{2}^{N_{\sigma(t)}\left(t_{0}, t\right)} \mu_{1}^{N_{\sigma^{\prime}(t)}\left(t_{0}, t\right)} \int_{t_{0}}^{t_{1}} e^{\beta T^{+}(s, t)-\alpha T^{-}(s, t)} \Gamma^{T}(s) \Gamma(s) \mathrm{d} s+\cdots \\
& +\mu_{2}^{N_{\sigma(t)}\left(t_{k-1}, t\right)} \mu_{1}^{N_{\sigma^{\prime}(t)}\left(t_{k-1}+\Delta_{k-1}, t\right)} \int_{t_{k-1}+\Delta_{k-1}}^{t} e^{\beta T^{+}(s, t)-\alpha T^{-}(s, t)} \Gamma^{T}(s) \Gamma(s) \mathrm{d} s \\
& +\mu_{2}^{N_{\sigma(t)}\left(t_{k}, t\right)} \mu_{1}^{N_{\sigma^{\prime}(t)}\left(t_{k-1}+\Delta_{k-1}, t\right)} \int_{t_{k}}^{t} e^{\beta T^{+}(s, t)-\alpha T^{-}(s, t)} \Gamma^{T}(s) \Gamma(s) \mathrm{d} s \\
& =\mu_{2}^{N_{\sigma(t)}\left(t_{0}, t\right)} \mu_{1}^{N_{\sigma^{\prime}(t)}\left(t_{0}, t\right)} V_{\sigma\left(t_{0}\right)}\left(t_{0}\right) e^{\beta T^{+}\left(t_{0}, t\right)-\alpha T^{-}\left(t_{0}, t\right)} \\
& +\int_{t_{0}}^{t} \mu_{2}^{N_{\sigma(t)}(s, t)} \mu_{1}^{N_{\sigma^{\prime}(t)}(s, t)} e^{\beta T^{+}(s, t)-\alpha T^{-}(s, t)} \Gamma^{T}(s) \Gamma(s) \mathrm{d} s
\end{aligned}
$$

Under the initial condition $x\left(t_{0}\right)=0$, we obtain

$$
\begin{aligned}
V(t) & \leqslant \int_{t_{0}}^{t} \mu_{2}^{N_{\sigma(t)}(s, t)} \mu_{1}^{N_{\sigma^{\prime}(t)}(s, t)} e^{\beta T^{+}(s, t)-\alpha T^{-}(s, t)} \Gamma^{T}(s) \Gamma(s) \mathrm{d} s \\
& =\int_{t_{0}}^{t} e^{N_{\sigma(t)}(s, t) \ln \mu_{2}+N_{\sigma^{\prime}(t)}(s, t) \ln \mu_{1}} e^{\beta T^{+}(s, t)-\alpha T^{-}(s, t)} \Gamma^{T}(s) \Gamma(s) \mathrm{d} s
\end{aligned}
$$


Multiplying both sides of (35) by $e^{-\left[N_{\sigma(t)}\left(t_{0}, t\right) \ln \mu_{2}+N_{\sigma^{\prime}(t)}\left(t_{0}, t\right) \ln \mu_{1}\right]}$ yields

$$
\begin{aligned}
& e^{-\left[N_{\sigma(t)}\left(t_{0}, t\right) \ln \mu_{2}+N_{\sigma^{\prime}(t)}\left(t_{0}, t\right) \ln \mu_{1}\right]} V(t) \\
& \quad \leqslant \int_{t_{0}}^{t} e^{N_{\sigma(t)}\left(t_{0}, s\right) \ln \mu_{2}+N_{\sigma^{\prime}(t)}\left(t_{0}, s\right) \ln \mu_{1}} e^{\beta T^{+}(s, t)-\alpha T^{-}(s, t)} \Gamma^{T}(s) \Gamma(s) \mathrm{d} s
\end{aligned}
$$

When $t \in\left[t_{k}, t_{k}+\Delta_{k}\right), k=1,2,3, \ldots$, by the condition in (12), we have

$$
\begin{aligned}
-\left[N_{\sigma(t)}\left(t_{0}, t\right) \ln \mu_{2}+N_{\sigma^{\prime}(t)}\left(t_{0}, t\right) \ln \mu_{1}\right] & =-\left[N_{\sigma(t)}\left(t_{0}, t\right)\left(\ln \mu_{2}+\ln \mu_{1}\right)-\ln \mu_{1}\right] \\
& \geqslant-\left[\frac{t-t_{0}}{\tau_{a}^{*}} \ln \left(\mu_{1} \mu_{2}\right)-\ln \mu_{1}\right] \\
& =-\zeta^{*}\left(t-t_{0}\right)+\ln \mu_{1}
\end{aligned}
$$

By (27), (36), and (37), we have

$$
\begin{aligned}
e^{-\zeta^{*}\left(t-t_{0}\right)+\ln \mu_{1}} V(t) & \leqslant e^{-\left[N_{\sigma(t)}\left(t_{0}, t\right) \ln \mu_{2}+N_{\sigma^{\prime}(t)}\left(t_{0}, t\right) \ln \mu_{1}\right]} V(t) \\
& \leqslant \int_{t_{0}}^{t} e^{N_{\sigma(t)}\left(t_{0}, s\right) \ln \mu_{2}+N_{\sigma^{\prime}(t)}\left(t_{0}, s\right) \ln \mu_{1}} e^{-\zeta^{*}(t-s)} \Gamma^{T}(s) \Gamma(s) \mathrm{d} s \\
& \leqslant \int_{t_{0}}^{t} \Gamma^{T}(s) \Gamma(s) \mathrm{d} s
\end{aligned}
$$

which means

$$
\int_{t_{0}}^{t} e^{T}(s) e(s) \mathrm{d} s \leqslant \gamma^{2} \int_{t_{0}}^{t} \omega^{T}(s) \omega(s) \mathrm{d} s
$$

when $t \rightarrow \infty$, then the proof is completed.

\subsection{Fault detection filter design}

\section{Theorem 2}

Given constants $\alpha>0, \beta>0, \mu_{1} \geqslant 1$, and $\mu_{2} \geqslant 1$, if there exist positive-definite matrices $X_{i}, R_{i}$, $P_{22 i}, P_{11 i j}, P_{22 i j}$, and any matrices $P_{12 i}, P_{12 i j}, \bar{A}_{f i}, \bar{B}_{f i}, \bar{C}_{f i}$, and $\bar{D}_{f i}$, for $i \neq j, i, j \in N$, such that

$$
\begin{aligned}
& {\left[\begin{array}{ll}
P_{11 i j} & P_{12 i j} \\
P_{12 i j}^{T} & P_{22 i j}
\end{array}\right]>0, \quad\left[\begin{array}{cc}
X_{i} & P_{12 i} \\
P_{12 i}^{T} & P_{22 i}
\end{array}\right]>0} \\
& {\left[\begin{array}{cc}
X_{j} & P_{12 j} \\
P_{12 j}^{T} & P_{22 j}
\end{array}\right] \leqslant \mu_{1}\left[\begin{array}{cc}
P_{11 i j} & P_{12 i j} \\
P_{12 i j}^{T} & P_{22 i j}
\end{array}\right], \quad\left[\begin{array}{cc}
P_{11 i j} & P_{12 i j} \\
P_{12 i j}^{T} & P_{22 i j}
\end{array}\right] \leqslant \mu_{2}\left[\begin{array}{cc}
X_{i} & P_{12 i} \\
P_{12 i}^{T} & P_{22 i}
\end{array}\right]} \\
& {\left[\begin{array}{ccccc}
\varphi_{11} & \varphi_{12} & R_{i} B_{1 i} & R_{i} D_{1 i} & C_{i}^{T} \bar{D}_{f i}^{T}+\bar{C}_{f i}^{T} \\
* & \varphi_{22} & X_{i} B_{1 i}+\bar{B}_{f i} B_{2 i} & X_{i} D_{1 i}+\bar{B}_{f i} D_{2 i} & C_{i}^{T} \bar{D}_{f i}^{T} \\
* & * & -\gamma^{2} I & 0 & B_{2 i}^{T} \bar{D}_{f i}^{T} \\
* & * & * & -\gamma^{2} I & D_{2 i}^{T} \bar{D}_{f i}^{T}-I \\
* & * & * & * & -I
\end{array}\right]<0} \\
& {\left[\begin{array}{ccccc}
\phi_{11} & \phi_{12} & \phi_{13} & \phi_{14} & C_{j}^{T} D_{f i}^{T} \\
* & \phi_{22} & \phi_{23} & \phi_{24} & C_{f i}^{T} \\
* & * & -\gamma^{2} I & 0 & B_{2 j}^{T} D_{f i}^{T} \\
* & * & * & -\gamma^{2} I & D_{2 j}^{T} D_{f i}^{T}-I \\
* & * & * & * & -I
\end{array}\right]<0}
\end{aligned}
$$


where

$$
\begin{aligned}
& \varphi_{11}=R_{i} A_{i}+A_{i}^{T} R_{i}+\alpha R_{i} ; \\
& \varphi_{12}=R_{i} A_{i}+A_{i}^{T} X_{i}+C_{i}^{T} \bar{B}_{f i}^{T}+\bar{A}_{f i}^{T}+\alpha R_{i} ; \\
& \varphi_{22}=X_{i} A_{i}+A_{i}^{T} X_{i}+\bar{B}_{f i} C_{i}+C_{i}^{T} \bar{B}_{f i}^{T}+\alpha X_{i} ; \\
& \phi_{11}=P_{11 i j} A_{j}+P_{12 i j} B_{f i} C_{j}+A_{j}^{T} P_{11 i j}+C_{j}^{T} B_{f i}^{T} P_{12 i j}^{T}-\beta P_{11 i j} ; \\
& \phi_{12}=P_{12 i j} A_{f i}+A_{j}^{T} P_{12 i j}+C_{j}^{T} B_{f i}^{T} P_{22 i j}^{T}-\beta P_{12 i j} ; \\
& \phi_{13}=P_{11 i j} B_{1 j}+P_{12 i j} B_{f i} B_{2 j} ; \\
& \phi_{14}=P_{11 i j} D_{1 j}+P_{12 i j} B_{f i} D_{2 j} ; \\
& \phi_{22}=P_{22 i j} A_{f i}+A_{f i}^{T} P_{22 i j}-\beta P_{22 i j} ; \\
& \phi_{23}=P_{12 i j}^{T} B_{1 j}+P_{22 i j} B_{f i} B_{2 j} ; \\
& \phi_{24}=P_{12 i j}^{T} D_{1 j}+P_{22 i j} B_{f i} D_{2 j} .
\end{aligned}
$$

then there exits a fault detection filter in the form of (2), such that the residual error system $\Sigma\left(\right.$ or $\left.\Sigma^{\prime}\right)$ is asymptotically stable with $H_{\infty}$ performance $\gamma$ for any switching signal with average dwell time satisfying (12). Moreover, the desired fault detection filter realization is given by

$$
A_{f i}=P_{12 i}^{-1} \bar{A}_{f i}^{T} R_{i}^{-1} S_{12 i}^{-T}, \quad B_{f i}=P_{12 i}^{-1} \bar{B}_{f i}, \quad C_{f i}=\bar{C}_{f i}^{T} R_{i}^{-1} S_{12 i}^{-T}, \quad D_{f i}=\bar{D}_{f i} .
$$

where the matrices $P_{12 i}$ and $S_{12 i}$ satisfy $X_{i} R_{i}^{-1}+P_{12 i} S_{12 i}^{T}=I, P_{12 i}^{T} R_{i}^{-1}+P_{22 i} S_{12 i}^{T}=0$.

Proof

Let the positive-definite matrices $P_{i}$ and $P_{i}^{-1}$ be partitioned as

$$
P_{i}=\left[\begin{array}{cc}
P_{11 i} & P_{12 i} \\
P_{12 i}^{T} & P_{22 i}
\end{array}\right], \quad P_{i}^{-1}=\left[\begin{array}{cc}
S_{11 i} & S_{12 i} \\
S_{12 i}^{T} & S_{22 i}
\end{array}\right]
$$

By $P_{i} P_{i}^{-1}=I$, one can have that

$$
X_{i} R_{i}^{-1}+P_{12 i} S_{12 i}^{T}=I, \quad P_{12 i}^{T} R_{i}^{-1}+P_{22 i} S_{12 i}^{T}=0
$$

Define the following matrix

$$
J_{i}=\left[\begin{array}{ll}
S_{11 i} & I \\
S_{12 i}^{T} & 0
\end{array}\right]
$$

then pre-multiply and post-multiply $\operatorname{diag}\left\{J^{T}, I, I\right\}$ to (10), one obtains

$$
\left[\begin{array}{ccccc}
(1,1) & (1,2) & B_{1 i} & D_{1 i} & S_{11 i} C_{i}^{T} D_{f i}^{T}+S_{12 i} C_{f i}^{T} \\
* & (2,2) & P_{11 i} B_{1 i}+P_{12 i} B_{f i} B_{2 i} & P_{11 i} D_{1 i}+P_{12 i} B_{f i} D_{2 i} & C_{i}^{T} D_{f i}^{T} \\
* & * & -\gamma^{2} I & 0 & B_{2 j}^{T} D_{f i}^{T} \\
* & * & * & -\gamma^{2} I & D_{2 j}^{T} D_{f i}^{T}-I \\
* & * & * & * & -I
\end{array}\right]<0
$$

Pre-multiply and post-multiply $\operatorname{diag}\left\{S_{11 i}^{-1}, I, I, I, I\right\}$ to (48), and let $S_{11 i}^{-1}=R_{i}, P_{11 i}=X_{i}$. Combined with the condition in (44), one can have that (48) is equivalent to (42).

Similarly, the matrix $P_{i j}$ is also partitioned as

$$
P_{i j}=\left[\begin{array}{cc}
P_{11 i j} & P_{12 i j} \\
P_{12 i j}^{T} & P_{22 i j}
\end{array}\right]
$$

Substituting (5) and (49) to (11), one can obtain (40) and (43). Then, the proof is completed. 


\section{Remark 2}

Noting that the conditions in (40)-(44) are mutually dependent. Therefore, we can first solve (42) and (44) to obtain matrices $A_{f i}, B_{f i}, C_{f i}$, and $D_{f i}$. Then, the feasible solutions of $P_{11 i j}, P_{12 i j}$, and $P_{22 i j}$ can be found by solving(40), (41), and (43).

\section{Remark 3}

In practical operation, the condition in (12) is usually difficult to justify. We can assume that the maximum value of the lag $\Delta_{\max }$ between the fault detection filter and the original system is a known constant, then (12) can be reduced to the following condition

$$
\tau_{a}>\tau_{a}^{*}=\max \left\{\frac{\ln \left(\mu_{1} \mu_{2}\right)}{\zeta^{*}},\left(\frac{\beta+\zeta^{*}}{\alpha-\zeta^{*}}+1\right) \Delta_{\max }\right\}
$$

\section{Remark 4}

In this note, average dwell time approach is utilized to deal with fault detection problem. Compared with arbitrary switching and dwell time switching, average dwell time switching approach is less conservative.

\section{EXAMPLE}

Switched system is commonly used in practical engineering application. For example, the switched electrical circuit shown in Figure 2 is described with two switching modes [31]. In mode 1, the so-called 'on' time, Sw1 is closed and Sw2 is open. In mode 2, the so-called 'off' time, Sw1 is open and $\mathrm{Sw} 2$ is closed. In this system, Sw1 is often a bipolar transistor and Sw2 is a diode. Vc is used to denote the capacitor voltage equal to the output volt age delivered to the load R1, and I1 denotes the inductor current. During the 'on' time, the inductor current is also equal to the input source current. During the 'off' time, the input source current is zero.

Therefore, in this example, the mode number of system (1) is $N=2$, and the state-space matrices are as follows:

$$
\begin{aligned}
& A_{1}=\left[\begin{array}{cc}
-2 & 0 \\
0 & 0.5
\end{array}\right], A_{2}=\left[\begin{array}{cc}
0.5 & 0 \\
0 & -1
\end{array}\right], B_{11}=\left[\begin{array}{l}
2 \\
1
\end{array}\right], B_{12}=\left[\begin{array}{l}
2 \\
1
\end{array}\right], B_{21}=1, B_{22}=0.5, \\
& C_{1}=\left[\begin{array}{ll}
1 & 2
\end{array}\right], C_{2}=\left[\begin{array}{ll}
1 & 0.5
\end{array}\right], D_{11}=1, D_{12}=2, D_{21}=\left[\begin{array}{cc}
0.5 & -1
\end{array}\right], D_{22}=\left[\begin{array}{ll}
-2 & 1
\end{array}\right] .
\end{aligned}
$$

The aim is to design a switched fault detection filter such that the augmented system $\Sigma$ (or $\Sigma^{\prime}$ ) is asymptotically stable and the performance defined in (6) is guaranteed. Set $\alpha=0.7, \beta=5.2$, $\mu_{1}=1.4$, and $\mu_{2}=2$. By solving the conditions in Theorem 2 , we can obtain a set of solutions of

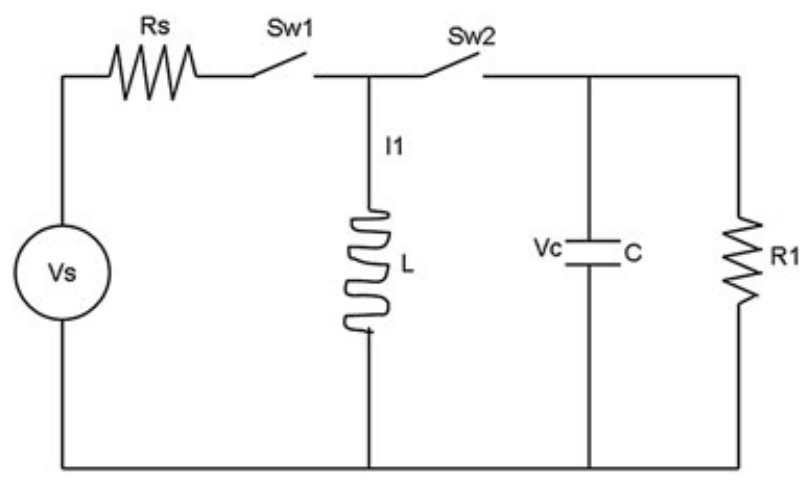

Figure 2. A switched electrical circuit. 
the fault detection filter parameters as follows:

$$
\begin{aligned}
& A_{f 1}=\left[\begin{array}{ll}
5.9745 & -2.5489 \\
0.2474 & 15.5439
\end{array}\right], B_{f 1}=\left[\begin{array}{r}
-7.1749 \\
0.3307
\end{array}\right], \\
& C_{f 1}=\left[\begin{array}{ll}
-19.0932 & -0.2363
\end{array}\right], D_{f 1}=3.4573 \\
& A_{f 2}=\left[\begin{array}{rr}
11.9462 & 0.1354 \\
6.0133 & 2.6065
\end{array}\right], B_{f 2}=\left[\begin{array}{l}
0.1671 \\
0.3191
\end{array}\right] \text {, } \\
& C_{f 2}=\left[\begin{array}{ll}
14.8903 & -2.7095
\end{array}\right], D_{f 2}=5.8041 .
\end{aligned}
$$

and the guaranteed performance defined in (6) is $\gamma=1.8$.

To demonstrate the effectiveness of the designed method, the disturbance $d(t)$ is assumed to be uniform random number, and the fault is set up as

$$
f(t)=\left\{\begin{array}{rc}
10, & 50 \leqslant t \leqslant 200 \\
0, & \text { others }
\end{array},\right.
$$

Once the fault occurs, the residual response $r(t)$ is depicted in Figure 3. Figure 4 presents the evolution of the residual evaluation function $J_{\mathrm{r}}(t)$ for both the faulty case (solid line) and fault-free case (dashed line). With a selected threshold $J_{\text {th }}=0.5982$ for $t=200$, the simulation results show that $J_{\mathrm{r}}(t)=0.6078>J_{\mathrm{th}}$ for $t=70$, which means that the fault $f(t)$ can be successfully detected about 20 time steps after its occurrence.

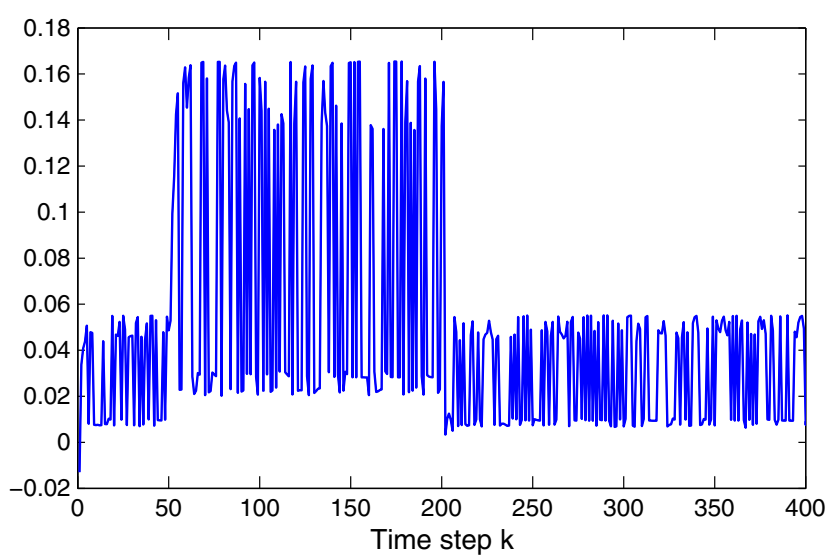

Figure 3. The residual response of $r(t)$.

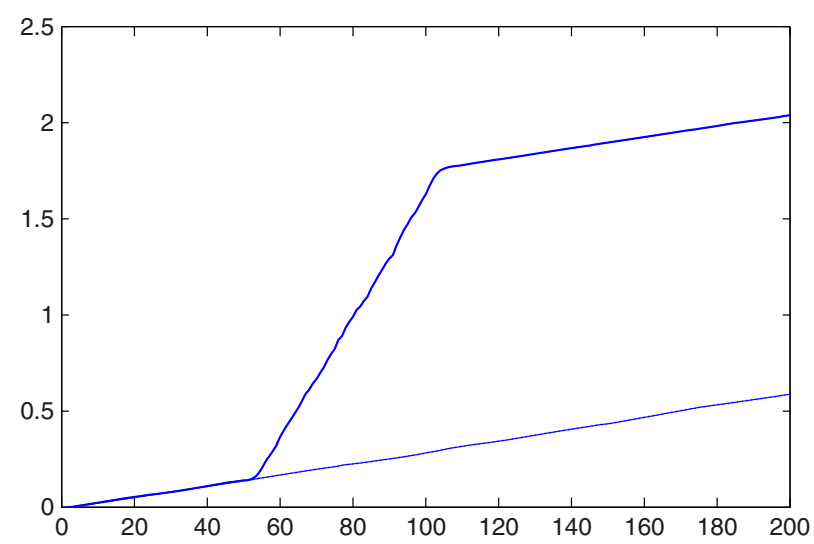

Figure 4. Evolution of residual evaluation function. 


\section{CONCLUSION}

In this paper, the problem of fault detection for continuous-time switched systems under asynchronous switching has been considered. The filter is assumed to be unmatched with the original system. An efficient condition has been given to construct the filter under the switching signal with average dwell time. Finally, an example has been provided to illustrate the proposed methods. On the basis of the obtained results in this note, fault estimation and accommodation for switched systems under asynchronous switching will be considered in the future work.

\section{ACKNOWLEDGEMENTS}

The authors wish to thank the editor and reviewers for their helpful and constructive comments and suggestions that have helped greatly improve the presentation of the paper.

This work was supported in part by the National Key Basic Research Program, China (2012CB215202), the 111 Project (B12018), the National Natural Science Foundation of China (61010121, 61034005, 61174058), Doctoral Fund of Ministry of Education of China (20113218110011), Funding for Outstanding Doctoral Dissertation in Nanjing University of Aeronautics and Astronautics (BCXJ10-03), Funding of Jiangsu Innovation Program for Graduate Education (CXZZ11-0214), and the Engineering and Physical Sciences Research Council, UK (EP/F029195).

\section{REFERENCES}

1. Frank PM, Ding X. Survey of robust residual generating and evaluation methods in observer-based fault detection systems. Journal of Process Control 1997; 7(6):403-424.

2. Hammouri H, Kinnaert M, El Yaagoubi EH. Observer based approach to fault detection and isolation for nonlinear systems. IEEE Transactions on Automatic Control 1999; 44(10):1879-1884.

3. White J, Speyer J. Detection filter design: Spectral theory and algorithms. IEEE Transactions on Automatic Control 1987; 32(6):593-603.

4. Chowdhury FN. Ordinary and neural chi-squared tests for fault detection in stochastic systems. IEEE Transactions on Control Systems Technology 2000; 8(2):372-379.

5. Ding SX, Zhong M, Jeinsch T, Tang B. LMI-based integration of robust H control and RFD for LTI systems. In Proceeding on IFAC World Congress, Barcelona, Spain, 2002; 494-499.

6. Chen W, Saif M. An iterative learning observer for fault detection and accommodation in nonlinear time-delay systems. International Journal of Robust and Nonlinear Control 2006; 16(1):1-19.

7. Tao G, Qi RY, Tan C. A parameter estimation based adaptive actuator failure compensation control scheme. Journal of Systems Engineering and Electronics 2011; 22(1):1-5.

8. Cai X, Wu F. Robust fault detection and isolation for parameter-dependent LFT systems. International Journal of Robust and Nonlinear Control 2010; 20(7):764-776.

9. Frank PM, Ding X, Koppen-Seliger B. Current developments in the theory of FDI. In Proceedings on IFAC Safeprocess, Budapest, Hungary, 2000; 16-27.

10. Chen J, Patton RJ. Robust Model-Based Fault Diagnosis for Dynamic Systems. Kluwer Academic Publishers: Norwell, MS, 1999.

11. Basseville M, Nikiforov I. Detection of Abrupt Changes-Theory and Applications. Prentice-Hall: Englewood Cliffs, NJ, 1993.

12. Zhong M, Ding S, Lam J, Wang H. An LMI approach to design robust fault detection filter for uncertain LTI systems. Automatica 2003; 39(3):543-550.

13. Zhao J, Hill DJ. Passivity and stability of switched systems: A multiple storate function method. Systems \& Control Letters 2008; 57(2):158-164.

14. Wang G, Liu Y, Wen C, Chen W. Delay-Dependent stability criterion and $H_{\infty}$ state-feedback control for uncertain discrete-time switched systems with time-varying delays. International Journal of Innovative Computing, Information and Control 2011; 7(5A):2473-2484.

15. Cui C, Long F, Li C. Disturbance attenuation for switched system with continuous-time and discrete-time subsystems: state feedback case. ICIC Express Letters 2010; 4(1):205-212.

16. Liu Y, Sun L, Lu B, Dai M. Feedback control of networked switched fuzzy time-delay systems based on observer. ICIC Express Letters 2010; 4(6B):2369-2376.

17. Zhang LX, Boukas E, Shi P. Exponential $H_{\infty}$ filtering for uncertain discrete-time switched linear systems with average dwell time: A $\mu$-dependent approach. International Journal of Robust and Nonlinear Control 2008; 18(11):1188-1207.

18. Zhang L, Gao HJ. Asynchronously switched control of switched linear systems with average dwell time. Automatica 2010; 46:953-958.

19. Zhang L, Jiang B. Stability of a class of switched linear systems with uncertainties and average dwell time switching. International Journal of Innovative Computing, Information and Control 2010; 6(2):667-676. 
20. Mahmoud MS, Xia Y. Robust filter design for piecewise discrete-time systems with time-varying delays. International Journal of Robust and Nonlinear Control 2010; 20(5):544-560.

21. Du D, Jiang B, Shi P, Zhou S. $H_{\infty}$ filtering of discrete-time switched systems with state delays via switched Lyapunov function approach. IEEE Transactions on Automatic Control 2007; 52(8):1520-1525.

22. Wang D, Wang W, Shi P. Robust fault detection for switched linear systems with state delays. IEEE Transactions on Systems, Man, and Cybernetics, Part B 2009; 39(3):800-805.

23. Zhang D, Wang H, Lu B, Wang Z. LMI-based fault detection fuzzy observer design with multiple performance constraints for a class of non-linear systems: comparative study. International Journal of Innovative Computing, Information and Control 2012; 8(1B):633-645.

24. Jiang B, Du D, Cocquempot V. Fault detection for discrete-time switched systems with interval time-varying delays. International Journal of Control, Automation and Systems 2011; 9(2):396-401.

25. Wang D, Shi P, Wang W. Robust fault detection for continuous-time switched delay systems: an linear matrix inequality approach. IET Control Theory Applications 2010; 4(1):100-108.

26. Du D, Jiang B, Shi P. Fault diagnosis for switched systems with time delay. The 8th IEEE International Conference on Control and Automation, Xiamen, June 9-11, 2010; 2162-2165.

27. Du D, Jiang B, Shi P. Active fault-tolerant control for switched systems with time delay. International Journal of Adaptive Control and Signal Processing 2011; 25:466-480.

28. Xiang Z, Chen Q. Robust reliable control for uncertain switched nonlinear systems with time delay under asynchrenous switching. Applied Mathematics and Computation 2010; 216:800-811.

29. Hespanha JP, Morse AS. Stability of switched systems with average dwell time. In Proceedings of the 38th Conference Decision Control, Phoenix, AZ, 1999; 2655-2660.

30. Tseng CS, Chen BS. $L_{\infty}$-gain fuzzy control for nonlinear dynamic systems with persistent bounded disturbance. Proceedings of the 13th IEEE International Conference on Fuzzy Systems, Budapest, Hungary, Luly, 2004; $783-788$.

31. Allison A, Abbott D. Some benefits of random variables in switched control systems. Microelectronics Journal 2000; 31(7):515-522. 